\title{
ARAUSIS: Sistema de simulação para manejo sustentável de florestas de Araucária
}

\author{
C $_{\text {arlos }}$ Roberto $_{\text {Anquetta }}{ }^{(1)}$
}

\begin{abstract}
RESUMO
Este artigo apresenta um modelo de simulação para o manejo sustentável de Florestas Naturais de Araucária. Para validar o modelo foram utilizados dados advindos de três parcelas permanentes de um hectare, instaladas em 1995 e remedidas anualmente, situadas na Estação Experimental de São João do Triunfo, Centro-Sul do estado do Paraná. Informações sobre taxas de recrutamento, crescimento em diâmetro e mortalidade foram empregadas para o estudo, considerando a população de árvores com DAP acima de $10 \mathrm{~cm}$. O modelo de simulação fundamentou-se na metodologia de matriz de transição, a qual considera um vetor de freqüências por classe de DAP inicial., probabilidade de transição de uma classe para outra e mortalidade para cada classe de diâmetro. Dez classes foram empregadas no estudo, considerando duas populações distintas: todas as espécies em conjunto (floresta) e a araucária em separado. Simulações feitas com o modelo para avaliar efeitos de práticas de manejo na floresta. Os resultados obtidos na simulação, apesar de preliminares, indicaram que as práticas de manejo permitidas pelos órgãos ambientais precisam ser revistas. Cortes seletivos de baixa magnitude em pequenos fragmentos florestais, como os que são geralmente praticados e permitidos por lei, não favorecem a regeneração natural do pinheiro, a principal espécie dentro da estrutura da floresta. O simulador ARAUSIS, imitando o que ocorre na natureza, indicou que é necessária a existência de perturbações mais severas e abertura de grandes clareiras na floresta para promover a regeneração natural do pinheiro.
\end{abstract}

Palavras-chave: Floresta Ombrófila Mista, floresta natural, manejo florestal

\begin{abstract}
ARAUSIS: simulation system for sustainable management of araucaria forests. This article presents a simulation model for the sustainable management of Araucaria Natural Forests. To validate the model, data coming
\end{abstract}

\footnotetext{
${ }^{1}$ Eng $^{\mathrm{o}}$ Florestal, M.Sc., Ph.D., Professor do Departamento de Ciências Florestais da UFPR, Bolsista do $\mathrm{CNPq}$
} 
from three permanent plots of one hectare each were used. The plots were established in 1995 in the Experimental Station at São João do Triunfo, Paraná State, and have been remeasured annually since then to provide figures on recruitment rates, tree diameter growth and mortality for individuals over 10 $\mathrm{cm}$ DBH. The simulation model was based on the transition matrix method, which considers a vector of initial frequencies by $\mathrm{DBH}$ class, transition probabilities of a class for other, and mortality, as well. Ten classes were used in this study, considering two different populations: all the species together (forest) and araucaria in separate. Model simulations were carried out to evaluate effects of logging practices in the forest. The results from simulations, despite of being preliminary ones, indicated that the management practices allowed by the environmental official institution in Paraná need to be reviewed. Selective logging of low magnitude in small forest fragments, as those that are generally practiced and allowed by the law, do not favor the natural regeneration of araucaria, the main species occurring in the forest. ARAUSIS simulator, imitating what happens in the reality, indicated that is necessary the existence of more severe disturbances and large openings in the forest to promote the natural regeneration of araucaria.

Key-words: Araucaria forest, natural forest, forest management

\section{INTRODUÇÃO}

A Floresta com Araucária cobria originalmente cerca de $200.000 \mathrm{~km}^{2}$ em todo o Brasil, ocorrendo no Paraná (40\% de sua superfície), Santa Catarina (31\%) e Rio Grande do Sul (25\%) e em manchas esparsas no sul de São Paulo (3\%), internando-se até o sul de Minas Gerais e Rio de Janeiro (1\%) (CARVALHO, 1994). Contudo, a colonização para fins agropecuários e o extrativismo da madeira, iniciados a partir de 1895, conduziram à destruição de extensas áreas dessa formação florestal. Nessa época, o Paraná dispunha de cerca de cerca de $73.780 \mathrm{~km}^{2}$ com floresta natural de pinheiro (MAACK, 1968).

Ao longo do processo histórico de ocupação do Paraná, assistiu-se a uma rápida eliminação de sua cobertura florestal (SONDA, 1996). Ao final da década de 70, apenas $3.166 \mathrm{~km}^{2}$ dos pinhais nativos haviam restado, ou seja, cerca de 4,3\% da área original. De acordo com a última informação oficial, a área de Floresta com Araucária no Paraná foi reduzida, em 1980, para 2.696 $\mathrm{km}^{2}$ (IBDF, 1984). Embora não existam dados oficiais recentes, acredita-se que na última década a situação deve ter se agravado ainda mais.

Conservar os remanescentes da Floresta com Araucária é um grande desafio para o Paraná e todo o sul do Brasil. A preservação integral dos fragmentos florestais por força da lei e da fiscalização não é por si só capaz de manter em quantidade e qualidade o que resta. Desestruturação e perda da 
diversidade das florestas remanescentes em toda a região Centro-Sul do Paraná são indicadores incontestáveis da ineficácia da política de licenciamento ambiental que hoje se impõe. Uma forma de conservar os remanescentes da Floresta com Araucária é estimular o seu manejo em bases sustentáveis, com geração e difusão de tecnologia. Lamentavelmente, até então, pouquíssima atenção foi dada à obtenção, interpretação e aplicação dos elementos de monitoração da produtividade primária, da dinâmica de populações, do crescimento, das taxas de regeneração natural, da mortalidade, entre outros, que deveriam ser proporcionados pelos inventários realizados obrigatoriamente quando da submissão de Planos de Manejo Florestal aos órgãos oficiais.

Este artigo tem a finalidade de introduzir um sistema informatizado de simulação dos processos dinâmicos (crescimento, mortalidade e regeneração natural) de Florestas com Araucária. O sistema, chamado ARAUSIS, permite, simular o desenvolvimento da floresta, com base em dados de inventário florestal convencional, possibilitando analisar o efeito de diferentes práticas de manejo conduzidas na área em apreço.

\section{MATERIAL E MÉTODOS}

O sistema foi calibrado utilizando dados obtidos na Estação Experimental de São João do Triunfo, de propriedade da Universidade Federal do Paraná, a qual situa-se a cerca de $125 \mathrm{~km}$ de Curitiba. A área da Estação totaliza 32 ha, sua altitude é de $780 \mathrm{~m}$ e suas coordenadas geográficas são as seguintes: $25^{\circ} 34^{\prime} 18^{\prime \prime}$ latitude Sul e 50 $05^{\prime} 56^{\prime \prime}$ longitude Oeste de Greenwich. O clima da região é classificado como Cfb e o solo predominante é o podzólico vermelho-amarelo distrófico, com pequena porção de cambissolo distrófico álico (LONGHI, 1980). A vegetação caracteriza-se como um fragmento de Floresta Ombrófila Mista ou Floresta com Araucária, tendo como predomintes o pinheiro-brasileiro (Araucaria angustifolia) e diversas espécies folhosas, como a imbuia (Ocotea porosa), erva-mate (Ilex paraguariensis), canelas (Lauraceae), membros da família Myrtaceae, entre outras. A floresta sofreu intervenções antrópicas no passado, porém ainda mantém estrutura e composição florística típicas de florestas pouco alteradas.

Os dados empregados no desenvolvimento do sistema advêm de três unidades de um 1 ha cada $(100 \times 100 \mathrm{~m})$ e outra de forma irregular com 0,5 ha, instaladas em junho de 1995 e remedidas anualmente desde então. Nas parcelas, toda árvore com DAP $>10 \mathrm{~cm}$ foi pintada, etiquetada, posicionada em escala em um croqui, medida em CAP e identificada botanicamente. 


\section{RESULTADOS E DISCUSSÃo}

\section{Características do sistema}

O simulador do crescimento e da produção de Floresta com Araucária, denominado ARAUSIS, é um sistema de equações fundamentado no modelo matricial de transição para prognose da estrutura e da produtividade primária da floresta.

O modelo matricial de transição, para efetivar prognose de produção, tem-se o volume inicial da floresta, por exemplo, no momento " $\mathrm{t}$ " (presente) que se conhece, e o volume a ser prognosticado num certo momento temporal "t+p" (futuro). Conforme demonstrado por SANQUETTA et al. (1996), para se obter a prognose volumétrica é necessário efetuar a prognose do número de árvores, a qual é dada por:

$$
\mathrm{N}_{\mathrm{t}+\mathrm{p}}=\mathrm{T}_{\mathrm{p}} \cdot \mathrm{N}_{\mathrm{t}}+\mathrm{R}_{\mathrm{p}}
$$

onde:

$\mathrm{N}_{\mathrm{t}+\mathrm{p}}=$ vetor do número de árvores em classes diamétricas no ano " $\mathrm{t}+\mathrm{p}$ ";

$\mathrm{N}_{\mathrm{t}}=$ vetor do número de árvores em classes diamétricas no ano " $\mathrm{t}$ ";

$\mathrm{T}_{\mathrm{p}}=$ matriz probabilística de transição da floresta para o período " $\mathrm{p}$ ";

$\mathrm{R}_{\mathrm{p}}=$ vetor de recrutamento (ingresso) na floresta durante o período " $\mathrm{p}$ ".

A matriz de transição " $\mathrm{T}_{\mathrm{P}}$ ” tem a seguinte forma:
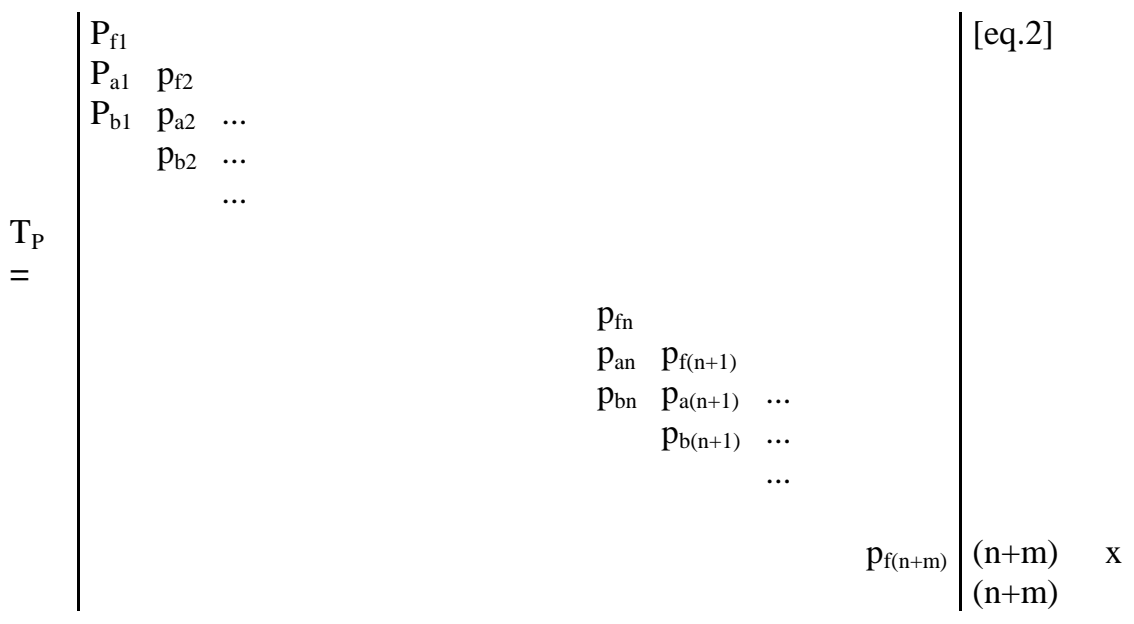

onde:

$\mathrm{p}_{\mathrm{fi}}=$ probabilidade que uma certa árvore de uma determinada classe de diâmetro permaneça na mesma classe de diâmetro "i" durante o período "p"; 
$\mathrm{p}_{\mathrm{ai}}=$ probabilidade que a planta avance para uma classe de diâmetro imediatamente maior durante o período " $p$ " considerado;

$\mathrm{p}_{\mathrm{bi}}=\mathrm{a}$ probabilidade que a planta avance duas classes de diâmetro durante o período considerado;

$\mathrm{n}=$ número de classes de diâmetro consideradas na matriz de transição e nos vetores de número de árvores (nesta pesquisa foram usadas 10 classes);

$\mathrm{m}=$ número de classes de diâmetro adicionadas até o período final da prognose (nesta pesquisa foram usadas 2 classes).

\section{Aplicações do sistema}

O sistema ARAUSIS foi aplicado na prática, em um estudo de caso, para avaliar o comportamento da floresta em termos de seus processos dinâmicos, ou seja, crescimento diamétrico, taxas de mortalidade e de recrutamento de diversas espécies nativas ocorrentes na Floresta Ombrófila Mista de São João do Triunfo-PR. As principais espécies contempladas nas simulações foram classificadas em quatro grupos produtivos, de acordo com critérios empregados pela indústria de madeira, isto é, pinheiro (Araucaria angustifolia), imbuia (Ocotea porosa), canelas (Lauraceae) e outras espécies ou madeiras brancas.

A partir do sistema foram gerados vetores de número de árvores por classe de DAP para cada grupo de espécies selecionado, bem como para o conjunto de todas as espécies, conforme ilustrado na Figura 1. Os vetores " $\mathrm{N}_{\mathrm{t}}$ ", preditos para momentos " $\mathrm{t}$ " no futuro, foram gerados também para analisar diversos cenários de cortes hipotéticos na floresta sob manejo, confrontando-os com o convencionalmente proposto em Planos de Manejo (corte seletivo de árvores com DAP>40 cm). A simulação incluiu a verificação da recuperação da capacidade produtiva da floresta em diversos ciclos de corte, após a realização de intervenções. Com algumas adaptações o sistema também permite que sejam avaliados simultaneamente os efeitos das intervenções madeireiras na biodiversidade das espécies lenhosas.

Os resultados obtidos na simulação, apesar de ainda serem parciais e preliminares, indicam que as práticas de manejo permitidas pelos órgãos ambientais precisam ser revistas. Cortes seletivos de baixa magnitude em pequenos fragmentos florestais, como os que são geralmente praticados e permitidos por lei, não favorecem a regeneração natural do pinheiro, a principal espécie dentro da estrutura da floresta. O simulador ARAUSIS, imitando o que ocorre na natureza, indicou que é necessária a existência de perturbações mais severas e abertura de grandes clareiras na floresta para promover a regeneração natural do pinheiro. Simultaneamente, para que a regeneração de fato se verifique, é imprescindível que haja um grande entorno florestado adjacente à área objeto das intervenções. Isto é necessário para que seja garantido o suprimento de quantidade grande de propágulos, especialmente no caso do pinheiro que tem suas sementes predadas por muitos animais e também 
consumidas pelo homem.

Os resultados parciais obtidos por simulação, apesar de ainda serem alvo de críticas e consistência, permitem antever a potencialidade do sistema ARAUSIS em gerar informações úteis aos elaboradores e executores de Planos de Manejo na Floresta com Araucária, bem como aos órgãos ambientais responsáveis pela sua aprovação. Poder-se-ia indicar que, a partir dos dados das parcelas permanentes, exigidas quando da elaboração do Plano de Manejo, e de outras características da área a ser manejada seria possível analisar as taxas de crescimento, mortalidade e recrutamento das espécies, bem como, a partir destas, decidir, em bases ecológicas e biométricas, o ciclo de corte apropriado para a floresta, os percentuais de corte de árvores por classes de diâmetro a serem permitidos por grupo de espécies, bem como eventuais tratamentos silviculturais a serem executados na floresta.

Figura 1 - Exemplos de distribuições diamétricas observadas e preditas pelo sistema ARAUSIS na Floresta de São João do Triunfo - PR (ano base 1995 - Floresta sem intervenção)

Figure 1 - Actual and predicted diameter distributions by ARAUSIS simulator for the forest at São João do Triunfo - PR (base year 1995 0-forest without logging)
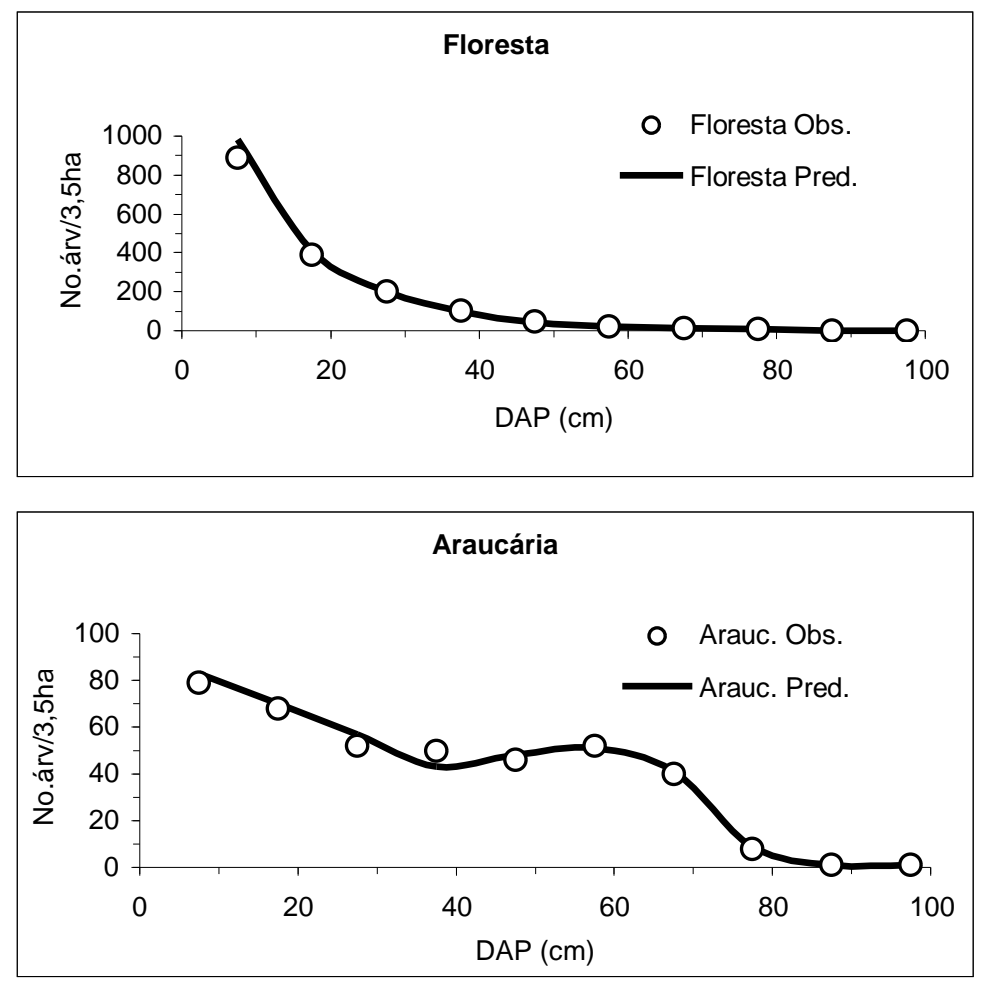


\section{BIBLIOGRAFIA CITADA}

CARVALHO, P.E.R. Espécies florestais brasileiras: recomendações silviculturais, potencialidades e uso da madeira. Empresa Brasileira de Pesquisa Agropecuária, Centro Nacional de Pesquisas Florestais. Colombo: EMBRAPA-CNPF; Brasília: EMBRAPA-SPI, 1994. 640p.

IBDF - INSTITUTO BRASILEIRO DE DESENVOLVIMENTO FLORESTAL. Inventário Nacional. Florestas Nativas - Paraná e Santa Catarina. Brasília, 1984. 345p.

LONGHI, S.J. A estrutura de uma floresta natural de Araucaria angustifolia (Bert.) O. Ktze, no sul do Brasil. Dissertação de Mestrado - PósGraduação em Engenharia Florestal - UFPR. Curitiba, 1980. 198p.

MAACK, R. Geografia física do Estado do Paraná. Curitiba: CODEPAR, 1968. 350p.

SANQUETTA, C. R.; ANGELO, H.; BRENA. D.A. \& MENDES, J.B. 1996. Predição da distribuição diamétrica, mortalidade e recrutamento de floresta natural com matriz Markoviana de potência. Floresta, v. 24, n. 1/2, p.2336, 1996.

SONDA, C. A floresta no Estado do Paraná: condicionantes naturais, econômicos e sociais. Dissertação de Mestrado. Universidade Técnica de Lisboa. Lisboa, 1996. 190p.

\section{AGRADECIMENTOS}

$\mathrm{O}$ autor agradece ao $\mathrm{CNPq}$ pelo suporte financeiro ao desenvolvimento desta pesquisa. Os agradecimentos são também estendidos a diversos professores, funcionários e acadêmicos do Curso de Engenharia Florestal da UFPR. 